\title{
Evaluación clínica del uso de nutrición enteral domiciliaria en pacientes pediátricos
}

\author{
Clinical evaluation in pediatric patients \\ with home enteral nutrition
}

\begin{abstract}
Enteral nutrition (EN) is the delivery of nutrients to the gastrointestinal tract by nasogastric (SNG), nasojejunal (SNY) or gastrostomy $(G T T)$, being the preferred route to fulfill nutritional needs when the oral route is contraindicated or inadequate. Objective: To describe the clinical profile of pediatric patients with home EN and to assess their nutritional status. Subjects and methods: A retrospective analytic study, in 37 children from the Pediatric Service Hospital Clinico UC, discharged with enteral nutrition support from April 2010 to April 2011. Results: The main indication to use $E N$ was swallowing disorder $(n=27)$. $73 \%$ began EN before one year of life. GTT was the principal route to EN $(n=20)$. There were no complications associated to NGT or OGT. The reported complications associated with GTT were periosteal granuloma $(n=7)$, aspiration pneumonia $(n=3)$ and accidental removal $(n=1) .62 \%$ of patients had protein-calorie malnutrition $(z$-score $W / H)$ at baseline vs. $30 \%$ at the last control $(p<0,001)$. Conclusion: The use of enteral nutrition allows to restore and/ or to maintain nutritional status in children with swallowing disorder or undernutrition. This feeding procedure also improves the quality of life and promotes a proper development without major complications.
\end{abstract}

Key words: Enteral nutrition, nutritional status, NGT, GTT, pediatric.

\section{INTRODUCCIÓN}

El soporte nutricional es un componente clave del cuidado médico. La nutrición enteral (NE) es la entrega de nutrientes al tracto gastrointestinal mediante una sonda nasogástrica (SNG), nasoyeyunal (SNY) o de gastrostomía (GTT); siendo la ruta preferida para satisfacer las necesidades nutricionales cuando la vía oral está contraindicada o es insuficiente y existe un tracto gastrointestinal funcionante (1). En las ultimas dos décadas, la indicación de nutrición enteral se ha incrementado de modo importante (2). El uso de nutrición enteral es un método seguro y eficaz en un momento de la vida en que los requerimientos nutricionales son altos (3).

Las principales indicaciones para nutrición enteral en pediatría son los trastornos motores y anatómicos del aparato digestivo, la aversión oral severa y la presencia de patologías emaciantes, crónicas o que requieran un alto aporte energético (3).

Cuando se requiere un apoyo nutricional por varios meses o años, la nutrición enteral domiciliaria (NED) es la mejor opción para los niños y sus familias. El interés por este tipo
Ana Cristina Tinoco O. (1)

Paola Pino A. (2)

Alejandro Zavala B. (3)

M. Isabel Hodgson B. (4)

(1) Facultad de Medicina, Pontificia Universidad Católica de Chile, Santiago, Chile (2) Servicio de Pediatría, Pontificia Universidad Católica de Chile, Santiago, Chile. (3) División de Cirugía, Unidad de Cirugía Pediátrica. Pontificia Universidad Católica de Chile, Santiago, Chile. (4) División de Pediatría, Unidad de Gastroenterología y Nutrición, Pontificia Universidad Católica de Chile, Santiago, Chile.

Dirigir la correspondencia a: Dra. M. Isabel Hodgson B. División de Pediatría Unidad de Gastroenterología y Nutrición Pontificia Universidad Católica de Chile. Santiago, Chile E-mail: hodgson@med.puc.cl

Este trabajo fue recibido el 25 de Enero de 2012, aceptado con modificaciones el 16 de Octubre de 2012 y aceptado para ser publicado el 15 de Enero de 2013.

de nutrición se ha incrementado rápidamente alrededor del mundo (4). La NED es una técnica segura, cuyo objetivo es mejorar la calidad de vida de los pacientes, permitiendo que se desarrollen en su entorno habitual, evitando hospitalizaciones, y reduciendo los costos sanitarios (5).

En los países desarrollados se está implementando cada vez más el uso de NED (6). En España existe evidencia del uso de nutrición enteral domiciliaria con una prevalencia de 113 casos por millón, en Estados Unidos 415 casos por millón y en Reino Unido de 500 casos por millón $(7,8)$. Sin embargo, en Chile, no existen estudios que reporten la prevalencia del uso de este tipo de nutrición en pacientes pediátricos.

El objetivo del presente estudio fue describir el perfil de un grupo de pacientes pediátricos que requirieron NE domiciliaria y su efecto en el estado nutricional.

\section{SUJETOS Y MÉTODO}

Estudio analítico retrospectivo, que describe las características clínicas y evalúa el estado nutricional de 37 niños hospitalizados en el Servicio de Pediatría del Hospital Clínico 
UC que fueron dados de alta con soporte nutricional enteral entre abril 2010 y abril 2011.

Los datos fueron recolectados de las fichas clínicas y del registro interno que lleva la enfermera encargada de educación, la que realiza un seguimiento telefónico en formal mensual, con el fin de dar continuidad a la educación realizada durante la hospitalización. Los datos para la evaluación del estado nutricional (peso y talla) se recolectaron de las fichas clínicas en el momento en que se indicó la nutrición enteral y en el último control médico realizado dentro del período de estudio, que en promedio corresponde a tres meses post alta hospitalaria.

Las variables estudiadas fueron: sexo, edad, y diagnóstico de base del paciente; indicación para iniciar el soporte nutricional y edad al inicio de la NE; tipo de dispositivo utilizado, tiempo de permanencia del dispositivo, y tipo de alimentación; complicaciones asociadas al uso de nutrición enteral; evaluación del estado nutricional al inicio de la NE y al último control; y comparación del estado nutricional al inicio de la NE versus al último control.

En cuanto al tipo de alimentacion enteral, ésta se categorizó en fórmulas lácteas infantiles o derivados lácteos más papillas (sopas mixtas fortificadas elaboradas en el hogar), fórmula polimérica comercial y fórmula polimérica comercial más papillas.

Los parámetros utilizados para la evaluación del estado nutricional en los menores de 6 años fueron el puntaje $z$ peso/talla $(\mathrm{P} / \mathrm{T})$ y el puntaje $\mathrm{z}$ talla/edad (T/E), considerando desnutrición un puntaje $z$ menor a 2 . En los mayores de 6 años $(n=3)$, el criterio de evaluación nutricional fue el Indice de Masa Corporal (IMC), catalogando como desnutrición un IMC menor al percentilo 5 .
Para el análisis de los datos se utilizó el Programa SPSS 17.0. Para los análisis descriptivos se realizó análisis de frecuencia, y medidas de tendencia central y dispersión. Para los análisis inferenciales, se utilizaron las pruebas de Mc Nemar y Wilcoxon Signed Ranks Test para muestras relacionadas, considerando un valor significativo un $p<0,05$.

\section{RESULTADOS}

Durante el período de seguimiento fueron estudiados 37 pacientes que cumplieron con los criterios de inclusión. Una leve mayoría fue de sexo masculino $(n=19)$, con una edad promedio de de $47 \pm 56$ meses, una mediana de 22 meses y un rango entre 2 y 217 meses. El diagnóstico de base más frecuente fue la enfermedad neurológica (tabla 1), existiendo un número considerable de pacientes que además requiere soporte ventilatorio $(n=12)$ y hospitalización domiciliaria $(n=11)$.

Las indicaciones para iniciar el soporte nutricional fueron: trastorno de la deglución $(n=27)$, mal incremento ponderal $(n=6)$ e indicación mixta $(n=4)$. La edad al inicio de la NE fue en promedio $24 \pm 50$ meses, con una mediana de 3 meses y un rango entre 0 y 216 meses, siendo la mayoría de los pacientes menores de 1 año al momento de recibir la indicación $(n=27)$.

Según el tipo de dispositivo utilizado, la mayoría de los pacientes fue dado de alta con GTT $(n=20)$, seguido de SNG $(n=16)$ y sonda orogástrica (SOG) $(n=1)$. El tiempo de permanencia del dispositivo fue en promedio $9 \pm 14$ meses, con una mediana de 4 meses y un rango entre 1 y 60 meses en los pacientes con SNG y SOG; y de $37 \pm 46$ meses, con una mediana de 17 meses y un rango entre 1 y 151 meses en los niños con GTT. El tipo de alimentación más frecuente fue fórmula láctea más papilla (tabla 2). La mayoría de ellos no

TABLA 1

Distribución porcentual según diagnóstico de base.

$\begin{array}{lcc}\text { Diagnóstico de base } & \text { Frecuencia } & \text { Porcentaje } \\ \text { Enfermedad neurológica } & 11 & 30 \% \\ \text { Enfermedad cardiovascular } & 7 & 19 \% \\ \text { Enfermedad neuromuscular } & 6 & 16 \% \\ \text { Defectos congénitos }^{\mathrm{a}} & 5 & 13 \% \\ \text { Otros diagnósticos }^{\mathrm{b}} & 8 & 22 \% \\ \text { Total } & 37 & 100 \%\end{array}$

aDefectos congénitos: Stuve Wiedeman, múltiples malformaciones, síndrome D-George, triploidia, Jarcho-Leving.

'Otros diagnósticos: Asociación Vacter, atresia de vías biliares, neumonía grave, teratoma inmaduro.

TABLA 2

Distribución porcentual según tipo de alimentación.

\begin{tabular}{lcc}
\hline Tipo de alimentación & Frecuencia & Porcentaje \\
Fórmula láctea más comida & 11 & $30 \%$ \\
Fórmula enteral polimérica & 10 & $27 \%$ \\
Fórmula enteral más comida & 9 & $24 \%$ \\
Fórmula láctea & 7 & $19 \%$ \\
Total & 37 & $100 \%$ \\
\hline
\end{tabular}


recibió alimentación por vía oral $(\mathrm{n}=28)$.

En relación a las complicaciones asociadas al uso de $\mathrm{NE}$, no las hubo asociadas al uso de SNG ni de SOG. Las complicaciones en los pacientes con GTT fueron: granuloma periostomal $(n=7)$, neumonía por aspiración $(n=3)$ y retiro accidental $(n=1)$.

La evaluación del estado nutricional al inicio de la NE y al último control se presenta en la tabla 3. Al realizar la comparación utilizando las variables categóricas: pacientes con desnutrición versus pacientes sin desnutrición; se encontró que la proporción de pacientes con desnutrición calóricoproteica al inicio de la NE (23/37) versus la proporción de pacientes con desnutrición al último control (11/37), presentó una diferencia significativa $(p<0,001)$.

Además, al comparar el estado nutricional al inicio de la $N E$ versus al último control, utilizando las variables numéricas: índice $\mathrm{P} / \mathrm{T}$, índice $\mathrm{T} / \mathrm{E}$ y puntaje $\mathrm{z}-\mathrm{IMC}$; se encontró una diferencia significativa en relación a $\mathrm{P} / \mathrm{T}$ en pacientes menores de 6 años ( $p<0.001)$, no así en relación a T/E en el total de pacientes (tabla 4).

En las tablas 5 y 6 se enumeran las características clínicas con los diagnósticos de base de los ninos con desnutrición y sobrepeso al último control. Los niños que permanecieron con desnutrición al último control padecían enfermedades crónicas asociadas a un mal incremento ponderal de larga data.

\section{DISCUSIÓN}

El uso de nutrición enteral domiciliaria en países desarrollados es cada vez más frecuente (9). Sin embargo, en Chile, no existen estudios que reporten la prevalencia del uso de este tipo de nutrición. La nutrición enteral domiciliaria ha demostrado ser un tratamiento eficaz para conseguir una recu- peración del estado nutricional, con una buena aceptabilidad por parte de los pacientes y sus padres, quienes refirien sentirse cómodos, más felices y con mayor libertad (10).

La principal indicación para la nutrición enteral domiciliaria en pediatría, son los pacientes con alteraciones neurológicas, trastornos motores del aparato digestivo, mucositis inducida por quimioterapia, reflujo severo y aversión oral severa. Se incluyen también aquellos pacientes con alteración en la absorción y digestión de nutrientes como en algunas enfermedades inflamatorias intestinales, fibrosis quística, enteritis, fistulas intestinales de bajo débito, diarrea con malabsorción. Otras indicaciones frecuentes para el uso de nutrición enteral son condiciones asociadas con mal incremento ponderal como cardiopatías, enfermedad hepática, enfermedad renal, enfermedad pulmonar crónica, anorexia (3); lo que coincide con el presente estudio en que la principal enfermedad de base de nuestros pacintes fue la enfermedad neurológica seguida por la enfermedad cardiovascular.

El tipo de alimentación utilizada, depende del dispositivo de alimentación y de la vía de administración (11). En este estudio, se emplearon fórmulas enterales poliméricas comerciales al inicio de la nutrición enteral y posteriormente fórmulas lácteas y sopas mixtas artesanales. Esto concuerda con otros estudios, en donde recalcan la importancia de utilizar regímenes de alimentación flexibles para la familia y con una composición nutricional adecuada para este tipo de pacientes, salvo en el caso de pacientes con desnutrición severa, reflujo severo, o usuarios de SNY que para su alimentación requieren el uso de bombas de infusión y fórmulas industrializadas $(4,12)$.

La población estudiada está compuesta principalmente

TABLA 3

Estado nutricional al inicio de la NE y al último control.

\begin{tabular}{|c|c|c|c|c|}
\hline \multirow[t]{2}{*}{ Estado nutricional } & \multicolumn{2}{|c|}{ Al inicio de la NE } & \multicolumn{2}{|c|}{ Al último control } \\
\hline & $\mathrm{N}$ & $\%$ & $\mathrm{~N}$ & $\%$ \\
\hline Desnutrición calórico-proteica & 23 & $62 \% *$ & 11 & $30 \% *$ \\
\hline Eutrófico & 11 & $30 \%$ & 18 & $48 \%$ \\
\hline Sobrepeso & 3 & $8 \%$ & 7 & $19 \%$ \\
\hline Obesidad & 0 & $0 \%$ & 1 & $3 \%$ \\
\hline Total & 37 & $100 \%$ & 37 & $100 \%$ \\
\hline
\end{tabular}

TABLA 4

Comparación del estado nutricional al inicio de la NE versus al último control.

\begin{tabular}{|c|c|c|c|c|c|}
\hline Z-score & $\mathrm{N}$ & Mediana & Mínimo & Máximo & Valor $\mathrm{P}$ \\
\hline $\mathrm{T} / \mathrm{E}$ al inicio de NE & 37 & $-1,20$ & $-5,40$ & 0,90 & 0.922 \\
\hline T/E al último control & 37 & $-1,40$ & $-3,60$ & 1,20 & 0.922 \\
\hline $\mathrm{P} / \mathrm{T}$ al inicio de NE & 34 & $-2,01$ & -5.00 & 1,90 & 0.000 \\
\hline $\mathrm{P} / \mathrm{T}$ al último control & 34 & -0.45 & $-3,37$ & 2,94 & 0.000 \\
\hline z-IMC al inicio de NE & 3 & $-0,11$ & $-5,03$ & 0,36 & 1.000 \\
\hline z-IMC al último control & 3 & $-0,23$ & $-4,76$ & 0,40 & 1.000 \\
\hline
\end{tabular}


por pacientes con enfermedades crónicas; destacando que 24\% de ellos requiere además soporte ventilatorio no invasivo. Los diagnósticos de base de los pacientes son similares a los encontrados en estudios previos y concordante con las indicaciones para el inicio de soporte nutricional $(3,10)$.

En cuanto al tipo de dispositivo utilizado, la GTT fue la vía más frecuente para administrar la nutrición enteral. Esto se debe a que los pacientes estudiados son portadores de enfermedades crónicas y requieren nutrición enteral por más de 4 meses $(1,13)$. Además, el uso de GTT ha evolucionado con el tiempo de acuerdo al advenimiento de nuevas técnicas quirúrgicas (4).

En relación a los días de permanencia del dispositivo, a pesar de la indicación de uso de sondas enterales por un período no mayor de 6 meses, en este estudio la SNG fue utilizada por un período de hasta 60 meses. Muchas veces es difícil convencer a los padres de la instalación de GTT como método definitivo de alimentación, dado que las sondas nasoenterales son generalmente bien toleradas. También existe un número de pacientes que debido a su pronóstico no pueden ser sometidos a procedimientos quirúrgicos, prefiriendose el uso de SNG.

Con respecto a las complicaciones asociadas, al igual que en estudios previos, no se encontraron complicaciones graves; salvo tres pacientes que presentaron neumonía por aspiración, los cuales tuvieron una evolución favorable (14).
Algunos pacientes presentaron complicaciones mecánicas asociadas al uso de GT, no así complicaciones metabólicas ni gastrointestinales. Lo anterior difiere de lo publicado por Gomez et al, quienes encontraron complicaciones gastrointestinales asociadas al uso de GT, como vómitos, estreñimiento y regurgitación, y complicaciones mecánicas asociadas al uso de SNG como obstrucción, rotura y desplazamiento (15).

En cuanto a la evaluación del estado nutricional, se encontró una evolución favorable. Al inicio de la nutrición enteral 23 niños (62\%) presentaban desnutrición calóricoproteica vs $11(30 \%)$ al último control ( $\mathrm{P} / \mathrm{T}$ en menores de 6 años), demostrando que la nutrición enteral es una buena alternativa para los niños con patologías crónicas asociadas a compromiso nutricional. Siete niños presentaron sobrepeso y uno obesidad al último control. Estos resultados son similares a los publicados por Kang y cols, quienes encontraron una mejoría de la prevalencia de desnutrición desde 73\% al inicio del procedimiento, hasta $35 \%$ al control (16). Se ha reportado que la talla es un predictor menos sensible para medir los resultados de soporte nutricional, lo cual concuerda con los resultados del presente estudio (17). Algunos pacientes neurológicos hipotónicos pueden desarrollar sobrepeso dado que su gasto calórico real es menor al gasto estimado acorde edad, talla y peso, estimación en la que se basa la prescripción de nutrición enteral.

Todos los padres recibieron educación en forma continua

\section{TABLA 5}

Características de los niños con desnutrición calórico-proteica al último control.

\begin{tabular}{|c|c|c|c|c|c|c|}
\hline $\mathrm{N}^{\circ}$ & $\begin{array}{c}\text { Edad } \\
\text { en meses }\end{array}$ & $\begin{array}{c}\text { Diagnóstico } \\
\text { de base }\end{array}$ & $\begin{array}{c}\text { Soporte } \\
\text { ventilatorio }\end{array}$ & Indicación & $\begin{array}{c}\text { Tipo de } \\
\text { dispositivo }\end{array}$ & $\begin{array}{c}\text { Tipo de } \\
\text { alimentación }\end{array}$ \\
\hline 1 & 217 & Enfermedad neurológica & No & Mal incremento ponderal & SNG & Fórmula enteral más comida \\
\hline 2 & 6 & Defectos congénitos & No & Trastorno deglución & SOG & Fórmula láctea \\
\hline 3 & 45 & Enfermedad neuromuscular & Sí & Trastorno deglución & $\mathrm{GTT}$ & Fórmula enteral más comida \\
\hline 4 & 132 & Enfermedad neuromuscular & Sí & Trastorno deglución & $\mathrm{GTT}$ & Fórmula láctea más comida \\
\hline 5 & 125 & Enfermedad neurológica & Sí & Trastorno deglución & GTT & Fórmula láctea más comida \\
\hline 6 & 14 & Otros diagnósticos & No & Mal incremento ponderal & SNG & Fórmula enteral \\
\hline 7 & 9 & Otros diagnósticos & No & Trastorno deglución & $\mathrm{GT}$ & Fórmula enteral \\
\hline 8 & 3 & Enfermedad cardiovascular & No & Mal incremento ponderal & SNG & Fórmula láctea \\
\hline 9 & 2 & Otros diagnósticos & No & Mal incremento ponderal & SNG & Fórmula láctea \\
\hline 10 & 5 & Enfermedad cardiovascular & Sí & Mal incremento ponderal & SNG & Fórmula láctea \\
\hline
\end{tabular}

TABLA 6

Características de los niños con sobrepeso y obesidad al último control.

\begin{tabular}{ccccccc}
\hline$N^{\circ}$ & $\begin{array}{c}\text { Edad } \\
\text { (meses })\end{array}$ & $\begin{array}{c}\text { Diagnóstico } \\
\text { de base }\end{array}$ & $\begin{array}{c}\text { Soporte } \\
\text { ventilatorio }\end{array}$ & Indicación & $\begin{array}{c}\text { Tipo de } \\
\text { dispositivo }\end{array}$ & $\begin{array}{c}\text { Tipo de } \\
\text { alimentación }\end{array}$ \\
1 & 10 & Enfermedad neurológica & No & Indicación mixta & SNG & Fórmula láctea más comida \\
2 & 26 & Enfermedad neurológica & No & Trastorno deglución & SNG & Fórmula láctea más comida \\
3 & 13 & Enfermedad cardiovascular & No & Indicación mixta & SNG & Fórmula láctea más comida \\
4 & 27 & Defectos congénitos & No & Trastorno deglución & GT & Fórmula enteral \\
5 & 33 & Enfermedad neurológica & No & Trastorno deglución & SNG & Fórmula enteral \\
6 & 61 & Enfermedad neurológica & Sí & Trastorno deglución & GT & Fórmula láctea más comida \\
\hline
\end{tabular}


durante la hospitalización con respecto a las indicaciones para el uso de NE, cuidados específicos de la vía de alimentación, modalidad de alimentación, administración de medicamentos, e instalación de la sonda o curación de la ostomía. En todos ellos se efectuó seguimiento por enfermera en forma mensual, vía telefónica, además de sus controles médicos periódicos.

En conclusión, los datos encontrados en nuestro estudio concuerdan con otros en que detallan que la NE ayuda a promover y mantener una ganancia de peso adecuada en este grupo de niños con enfermedades crónicas, sin asociarse a complicaciones graves. Este procedimiento permite continuar la recuperación del niño en un ambiente de familia; lo cual genera mayor satisfacción y mejora el apego de padres a hijos.

Es de importancia destacar que sólo 30\% de los pacientes estudiados contaba con hospitalización domiciliaria, programa que haría más fácil el control de la nutrición enteral. En el resto de los pacientes, el control está a cargo de los padres.

\section{RESUMEN}

Introducción: La nutrición enteral (NE) es la entrega de nutrientes al tracto gastrointestinal mediante una sonda nasogástrica (SNG), nasoyeyunal (SNY) o de gastrostomía (GTT); siendo la ruta preferida para satisfacer las necesidades nutricionales cuando la vía oral está contraindicada o es insuficiente. Objetivo: Describir el perfil de pacientes pediátricos que requirieron NE domiciliaria y su efecto en el estado nutritivo. Sujetos y Método: Estudio analítico retrospectivo, en 37 niños hospitalizados en el Servicio de Pediatría del Hospital Clínico Universidad Católica (UC) que fueron dados de alta con soporte nutricional enteral durante el período de Abril 2010 a Abril 2011. Resultados: La indicación principal para iniciar soporte nutricional fue trastorno de deglución $(n=27)$, siendo la mayoría de los pacientes menores de 1 año al momento de recibir la indicación $(n=27)$. El tipo de dispositivo más utilizado fue GTT $(n=20)$. No hubo complicaciones asociadas al uso de SNG. Las complicaciones asociadas al uso de GTT fueron: granuloma periostomal $(n=7)$, neumonía por aspiración $(n=3)$ y retiro accidental $(n=1)$. Del total de pacientes evaluados, un $62 \%$ presentaba desnutrición calórico-proteica al inicio de la $\mathrm{NE}$ versus $30 \%$ al último control $(p<0,001)$. Conclusión: El uso de soporte nutricional enteral permite recuperar y/o mantener el estado nutricional de los niños con trastornos de la deglución y alteraciones en la curva del crecimiento, mejora la calidad de vida, y presenta una tasa baja de complicaciones mayores.

Palabras clave: Nutrición enteral, evaluación clínica, estado nutricional, SNG, GT, pediatría.

Agradecimientos: A la Dra. Marcela Monge, Médico Jefe Servicio de Pediatría y al Dr. Andrés Castillo, Médico Jefe Unidad Paciente Crítico Pediátrica. Hospital Clínico UC; quienes facilitaron los recursos para la realización del estudio. Al Dr. Jaime Cerda, especialista en salud pública del Departamento de Salud Pública de la Pontificia Universidad Católica de Chile, quien colaboró en el análisis estadístico de los resultados.

\section{BIBLIOGRAFÍA}

1. Boullata J, Nieman Carney L, Guenter P. (edit). ASPEN: Enteral Nutrition Handbook. American Society for Parenteral and Enteral Nutrition. Silverspring, MD; 2010. p. 73-90; 267-300; 331-50.

2. Duggan $C$, et al. (edit) Nutrition in Pediatrics. 4th Edition. Hamilton, Ontario Canada. 2008. Capitulo IV: p. 766-75.

3. Serrano MS, Mannick EE. Consultation with the Specialist: Enteral Nutrition. Pediatr Rev 2003; 24: 417-23.

4. Daveluy W, Guimber D, Uhlen S, Lescut D, Michaud L, Turck D, Gottrand F. Dramatic Changes in Home-based Enteral Nutrition Practices in Children during an 11-year period. J Pediatr Gastroenterol Nutr 2006; 43 (2): 240-4.

5. Moreno Villares JM. La práctica de la nutrición artificial domiciliaria en Europa. Nutr Hosp 2004; 19 (2): 59-67.

6. Luengo Pérez $L M$, Chicharro ML, Cuerda C, García Luna PP, Rabassa Soler A, el al Grupo NADYA-SENPE. Registro de Nutrición Enteral Domiciliaria en España 2007. Nutr Hosp 2009; 24 (6): 655-60.

7. Planas Vilá $M$, et al, Ministerio de Sanidad y Consumo España. Guía de práctica clínica de nutrición enteral domiciliaria, 2008; p.14-23. Disponible en https://nadyasenpees.sserver.es/publicaciones/guiaprac.pdf

8. Scott C. Home to home: Important issues to be resolved. Clin Nutr Update 1988; 1: 8.

9. Holden CE, Puntis JW, Charlton CP, Booth IW. Nasogastrig feeding at home: acceptability and safety. Arch Dis Child 1991; 66: 148-51.

10. Payne-James JJ. Enteral nutrition. Eur J Gastroenterol Hepatol 1995; 7: 501-6.

11. Bott L, Husson MO, Guimber D, Michaud L, ArnaudBattandler F, Turck D, Gottrand F. Contamination of gastrostomy feeding systems in children in a home-based enteral nutrition program. J Pediatr Gastroenterol Nutr 2001; 33: 266-70.

12. Marchand V, Motil KJ; NASPGHAN Committee on Nutrition. Nutrition Support for Neurologically Impaired Children: A Clinical Report of the North American Society for Pediatric Gastroenterology, Hepatology, and Nutrition. J Pediatr Gastroenterol Nutr 2006; 43: 123-35.

13. Nasogastric and gastrostomy tube feeding for children being cared for in the community. Scotland, 2003. Disponible en internet: www.nhshealthquality.org

14. Puntis JW. Nutritional support at home and in the community. Arch Dis Child 2001; 84: 295-8.

15. Gomez Candela C, Cos Blanco A, García Luna PP, Pérez de la Cruz A, Iglesias Rosado C, Vazquez C, et al. Complicaciones de la nutrición enteral domiciliaria. Resultados de un estudio multicéntrico. Nutr Hosp 2003; 18 (3): 167-73.

16. Kang A, Zamora SA, Scott RB, Parsons HG. Catch-up Growth in Children Treated With Home Enteral Nutrition. Pediatrics 1998; 102: 951-5.

17. Walker SP, Golden $\mathrm{MH}$. Growth in length of children recovering from severe malnutrition. Eur J Clin Nutr 1988; 42: 395-404. 